\title{
Classes de quadras urbanas determinadas pelos níveis de ruídos
}

\author{
Classes of urban blocks established by noise levels
}

André B. D. Mendonça[ ${ }^{[0]}$, Marcia Thais Suriano ${ }^{[b]}$, Léa Cristina Lucas de Souza ${ }^{[c]}$, Eliane Viviani ${ }^{[d]}$

[a] Mestrando em Engenharia Urbana pelo Programa de Pós-Graduação em Engenharia Urbana da Universidade Federal de São Carlos (UFSCar), São Carlos, SP - Brasil, e-mail: andre_bressa@hotmail.com

[b] Doutora em Ecologia e Recursos Naturais pela Universidade Federal de São Carlos (UFSCar), São Carlos, SP - Brasil, e-mail: marcia_suriano@yahoo.com.br

[c] Doutora em Ciências da Engenharia Ambiental pela Universidade de São Paulo (USP), professora do Departamento de Engenharia Civil da Universidade Federal de São Carlos (UFSCar), São Carlos, SP - Brasil, e-mail: leacrist.ufscar@gmail.com

[d] Doutora em Engenharia de Transporte pela Universidade de São Paulo (USP), professora do Departamento de Engenharia Civil da Universidade de São Carlos (UFSCar), São Carlos, SP - Brasil, e-mail: eviviani@ufscar.br

\section{Resumo}

O objetivo deste trabalho é atribuir classes de diferenciação dos níveis de pressão sonora encontrados em quadras urbanas pertencentes a uma mesma zona de ocupação. Descreve-se, assim, o estado atual do ruído e identificam-se áreas com níveis sonoros acima dos permitidos pelas Normas Regulamentadoras. Para isso, o método utilizado compreende a delimitação de uma área de estudo pertencente a uma zona de ocupação induzida na cidade de São Carlos (SP). Nessa área são feitas coletas de dados de níveis de pressão sonora em horários de pico de fluxo de veículos, os quais servem de dados de entrada em um modelo de previsão e mapeamento do ruído. Examina-se a adequação entre os níveis mapeados e as normas vigentes para o conforto acústico humano e propõem-se classes de ruídos por quadras urbanas. Os resultados permitem evidenciar as quadras para as quais ações de controle devem ser tomadas para a garantia da qualidade acústica do local.

Palavras-chave: Mapeamento sonoro. Regulamentações acústicas. Tráfego de veículos. Quadras urbanas.

\section{Abstract}

This paper aims at attributing classes of urban blocks of a same induced occupation zone for the differentiation of the sound pressure level achieved on the surroundings of these urban blocks. The actual state of noise is described and areas with noise levels above the limits suggested by the Brazilian Standards Regulations are identified. Therefore, the applied method comprehends a delimitation of the study area in an occupation zone of São Carlos (SP). In this area, sound pressure levels are collected in peak hours of vehicular flow, which are used as data entry for an application of a prediction model and noise mapping. The mapped levels are counterexamined with the current regulations for human acoustical comfort and classes of urban blocks are proposed. 
The results allow highlighting the urban blocks for which control actions should be taken into account, in order to guarantee the local quality of the acoustical environment.

Keywords: Noise mapping. Regulation acoustic. Traffic flow. Urban blocks.

\section{Introdução}

A Organização das Nações Unidas (WHO, 2011) aponta que $51 \%$ da população mundial ocupa os espaços urbanos, com estimativas alarmantes de crescimento demográfico. No entanto, ao mesmo tempo que as cidades são centros de desenvolvimento, também são focos de geração de resíduos, poluição e problemas dos mais diversos tipos que afetam a integridade ambiental. Uma dessas questões diz respeito ao ruído ambiental e seu poder de interferir no meio ambiente e na saúde da população.

Dentre as diversas fontes de ruído, a do tráfego viário é a mais relacionada ao incômodo humano. Essa fonte de ruído depende das condições gerais que caracterizam os automóveis, como o porte dos veículos (leves, pesados ou motocicletas), o volume de tráfego e a velocidade, como também das condições das vias, como aclives e declives, e das condições de superfície, especialmente relacionadas à presença de buracos.

Somados às fontes de ruído, o ambiente sonoro urbano é influenciado pelo seu entorno, pela quantidade, localização e distância entre edifícios, pelo tráfego veicular, pela arborização, pelos materiais utilizados nas fachadas das edificações etc. Assim, conforme as formas físicas urbanas, os espectros sonoros podem sofrer difração, reflexão e absorção, o que pode contribuir para um maior desconforto em determinadas áreas (GUEDES; BERTOLI, 2005).

Essa problemática se torna mais evidente no caso brasileiro, quando o planejamento das cidades muitas vezes não leva em consideração as questões acústicas e não apresenta um efetivo programa de controle de ruídos.

O Brasil possui alguns dispositivos legais e normativos, como a Lei Federal n. 10.257/2001 (conhecida como Estatuto da Cidade), Resoluções Conama n. 001/1990 (sobre a emissão de ruído) (BRASIL, 1990a), n. 002/1990 (institui o Programa de Silêncio) (BRASIL, 1990b) e n. 020/1994 (institui o Selo Ruído) (BRASIL, 1994), ainda as normas téc- nicas da Associação Brasileira de Normas Técnicas (ABNT) NBR 10.151:2000 (Avaliação do ruído em áreas habitadas visando ao conforto da comunidade), a NBR 10.152:1987 (Níveis de ruídos para o conforto acústico) e a NBR 7.731:1983 (Guia para execução de serviços de medição de ruído aéreo e avaliação de seus efeitos sobre o homem). Porém, o mapeamento sonoro não é uma ferramenta amplamente difundida e, como consequência, esse instrumento de planejamento fica restrito e aplicado no âmbito acadêmico científico, como demonstram os trabalhos de Moraes et al. (2003) em Belém (PA), Pinto e Mardones (2009) no Rio de Janeiro (RJ), Costa e Lourenço (2010) em Sorocaba (SP), Cantieri et al. (2010) em Curitiba (PR) e Souza e Giunta (2011) em Bauru (SP). Apenas a cidade de Fortaleza, no estado do Ceará, apresenta um programa de mapeamento sonoro por meio da Secretaria do Meio Ambiente (SEMAM, 2011).

Diferentemente atuou a Comunidade Europeia, que, por meio de uma política ambiental - Directive 2002/49/EC - (EUROPEAN COMMUNITIES, 2002), implementou o mapeamento de ruído como uma ferramenta importante no planejamento urbano, a qual obriga que os municípios com mais de $250 \mathrm{mil}$ habitantes elaborem um mapeamento sonoro para o controle do ruído.

Os mapas de ruído permitem quantificar os níveis de pressão sonora e sua distribuição espacial, gerando uma informação visual do comportamento acústico do ruído ambiental em uma área geográfica, em determinado horário ou momento ao longo do dia. Essa ferramenta ainda proporciona a avaliação da exposição da população, a possibilidade de desenvolvimento de cenários futuros, a identificação de áreas de conflito e propostas de soluções (SANTOS; VALADO, 2004). Também contribui para o controle de evolução com o tempo e verificação de ações efetivas (MARDONES, 2009). Para Moraes (2007), a partir dos resultados encontrados, o mapeamento sonoro pode se destinar a identificar as zonas com maior ou menor grau de 
contaminação, para que se consiga criar planos de ação no combate ao ruído.

Considerando a escala de abordagem da contaminação sonora, o estudo da macrozona urbana em zonas de ocupação e recuperação, por se tratar de um primeiro nível de aproximação espacial para definição de diretrizes em um município, não é escala adequada para identificar pontos específicos que já apresentem características acima dos limites aceitáveis de conforto. No entanto, o estudo acaba por incidir nas propostas de zonas de ocupação induzida de alguns Planos Diretores de municípios brasileiros, onde o adensamento é acompanhado pelo aumento do fluxo de veículos. Assim, surgem conflitos entre o adensamento permitido e o nível de ruído recomendado por normas vigentes.

Por isso, existe uma necessidade de serem feitas análises em escalas mais detalhadas, que permitam diferenciar o grau de contaminação acústica em quadras urbanas pertencentes a uma mesma zona de ocupação. Descreve-se, assim, o estado atual do ruído e identificam-se áreas com níveis sonoros acima dos permitidos pelas Normas Regulamentadoras.

\section{Materiais e métodos}

\section{Área de estudo e caracterização física}

O município de São Carlos está localizado no centro geográfico do Estado de São Paulo, nas coordenadas de referência de $22^{\circ} 01^{\prime} \mathrm{S}$ de latitude e $47^{\circ} 54^{\prime} 0$ de longitude. A cidade possui população estimada em aproximadamente 222 mil habitantes (IBGE, 2010) e área total de 1.137,303 km², 6\% dos quais correspondem à área urbana, e $33 \mathrm{~km}^{2} \mathrm{de}$ área urbana ocupada.

Para a pesquisa, foi selecionada uma fração urbana localizada nas proximidades do Centro antigo dessa cidade, com ocupação de uso misto, como: construções residenciais, escolas, comércios e outros serviços.

Foram identificadas as seguintes características do entorno edificado: largura de via e passeios e suas respectivas declividades, altura das edificações, tipo de pavimentação da via, uso e ocupação do solo, altura de muros e distância entre as edificações. Esse levantamento foi realizado por meio de mapas cadastrais (disponibilizados pela Prefeitura
Municipal de São Carlos), visitas in loco e complementado por visualização no programa Google Earth $^{\circledR}$ (acessadas online).

Sobre a representação da área, elaborou-se uma malha de referência de $100 \times 100$ m, de forma que os nós da malha fossem distribuídos por ruas e avenidas coletoras de grande importância e consideradas de grande fluxo de veículos para os padrões da cidade. Essa operação resultou na determinação de 44 pontos para medição de níveis de pressão sonora (Figura 1).

\section{Coleta de dados}

Para cada ponto de medição, foi caracterizado e contabilizado o fluxo de tráfego, diferenciando-se a quantidade e a composição dos veículos em leves, pesados (ônibus e caminhões) e motocicletas.

As medições de níveis de pressão sonora foram realizadas em dias de semana (terças, quartas e quintas-feiras), evitando-se as situações atípicas geradas pelos fins de semana e feriados, e em horários de pico, ou seja, considerados de maior fluxo veicular ( $7 \mathrm{~h}$ às $8 \mathrm{~h}, 12 \mathrm{~h}$ às $13 \mathrm{~h}$ e $17 \mathrm{~h} 30$ às $18 \mathrm{~h} 30)$.

Como parâmetro de medição dos níveis de pressão sonora, foi considerado o Leq, correspondendo ao nível de pressão sonora equivalente, ponderado em "A", obtido pelo valor médio quadrático da pressão sonora e expresso pela Equação 1 (ABNT, 2000):

Leq $=10 \log 1 / \mathrm{n} \sum_{\mathrm{i}=0}^{\mathrm{n}} 10^{\mathrm{Li} / 10}$

(Equação 1)

Onde:

Li é o nível de pressão sonora em dB(A). n é o número total de leituras.

Para os registros dos níveis sonoros equivalentes (Leq), foi utilizado o equipamento Analyser 2270-L da Brüel \& Kjær, tipo Hand-Held, classificado como tipo 1 de precisão. Para minimizar interferências causadas por ventos, foi acoplado um protetor de vento ao microfone. 0 aparelho foi configurado com as especificações para medições externas de ruído ambiental, atendendo a todas as especificações metodológicas indicadas pelas NBR 10.151 (ABNT, 


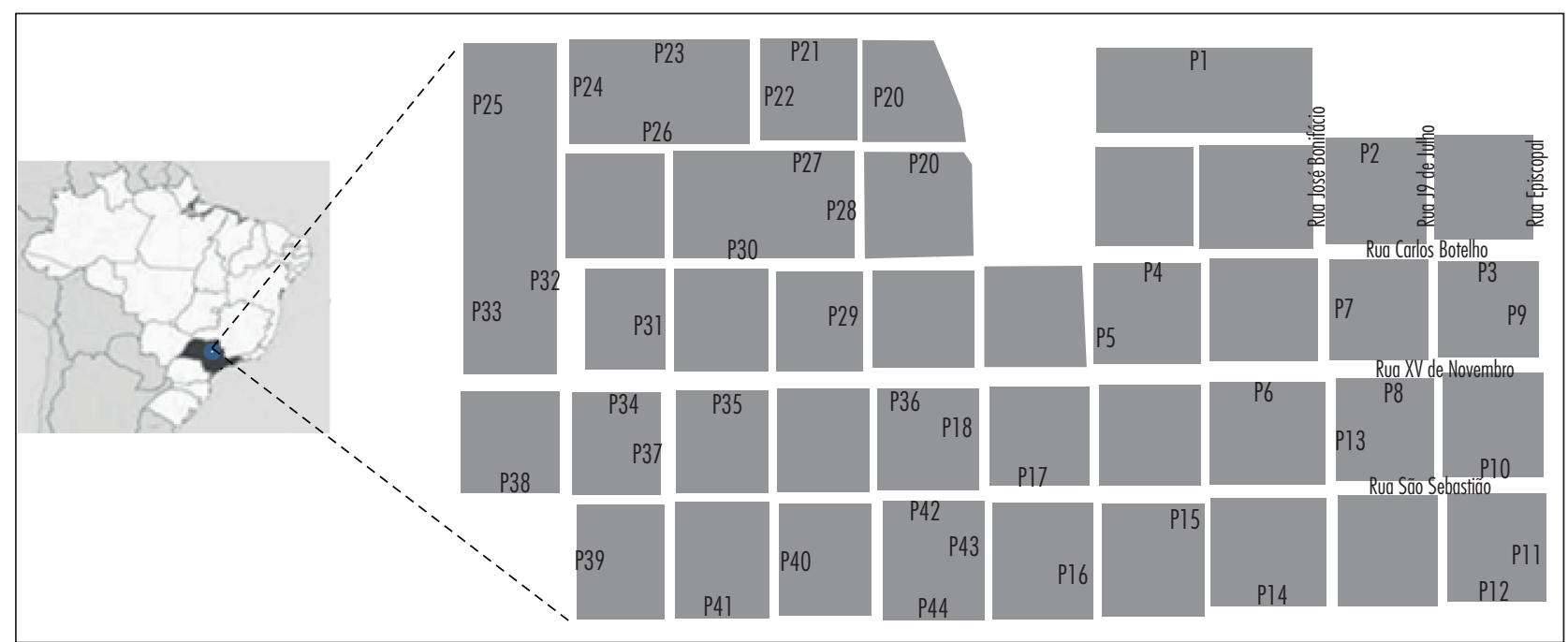

Figura 1 - Localização da cidade de São Carlos (São Paulo, Brasil), com destaque para área de estudo e os pontos de coleta (P) dos níveis sonoros Fonte: Dados da pesquisa.

2000) e ISO 1996. Utilizou-se o circuito de compensação em A com ponderação em tempo de resposta lenta (Slow). 0 aparelho foi afastado de superfícies refletoras, no mínimo $2 \mathrm{~m}$ de paredes e $1,2 \mathrm{~m}$ do chão, conforme a norma vigente.

Para determinação do tempo de duração das medições, foram considerados os resultados apontados por Mendonça et al. (2012). Naquele estudo, também para a cidade de São Carlos, os autores apontaram que, nos horários de pico de tráfego de veículos, a medição de Leq(A) durante 5 minutos é significativa e mantém forte correlação com níveis de Leq(A) medidos durante 15 minutos. Por isso, para otimização da coleta de dados, a duração de 5 minutos pode ser adotada, desde que para caracterização de horários de pico.

Aplicação dos dados em um modelo de previsão para o mapeamento acústico

A elaboração de mapas acústicos pode ser realizada sob três enfoques: medições dos níveis sonoros realizadas in loco e em pontos previamente determinados; simulações computacionais, utilizando modelos de predição acústica, como também a comparação entre as medições reais e as simulações (GUEDES; BERTOLI, 2005).

No caso aqui estudado, os dados de níveis de pressão sonora coletados foram incorporados no software Computer Aided Design Noise Abatement (CADNA-A) v. 4.1, da DATAKUSTIK (2005), com o intuito de calcular e apresentar os níveis de pressão sonora ambiental em forma de mapa, bem como prever e avaliar o incômodo acústico.

O CADNA-A, aplicado previamente em diversas pesquisas, como, por exemplo, Giunta (2013), Arana, R. San Martin e M. L. San Martin (2010) e Pinto e Mardones (2009), é considerado um programa capaz de calcular e apresentar os níveis de ruído ambiental, apresentando, sob a forma de mapas, intervalos de valores de ruído. 0 programa aplica normas internacionais para os cálculos, permitindo a especificidade de cada fonte sonora, seja ela rodovia, ferrovia, indústria ou qualquer fonte pontual ou linear.

Nele, as vias de tráfego são calculadas como fontes lineares divididas em segmentos que são assimilados pelo programa como uma fonte sonora pontual e que assume um nível de potência sonora, de acordo com as características do tráfego e da via (DATAKUSTIK, 2005).

Os dados de entrada de cada segmento podem ser relacionados à quantidade de veículos leves e pesados, à velocidade, à inclinação da via, ao tipo de pavimento e ao tipo de circulação. Ou, ainda, outra opção de entrada é inserir os dados de níveis sonoros reais coletados em pontos específicos, para obter os valores do mapa de ruído para toda a região de estudo. 
A partir das características atribuídas às fontes sonoras, o software considera que a trajetória direta da energia sonora entre a fonte e o receptor sofre atenuação devido à divergência geométrica, à absorção atmosférica, ao efeito do solo e à difração provocada pelos obstáculos. Além da trajetória direta, o software considera também o efeito das reflexões, ou seja, o efeito provocado pela trajetória indireta, pelo método da imagem refletida em um espelho (CARVALHO, 2006).

Nesse método, as superfícies refletoras são consideradas como espelhos nos quais as fontes sonoras produzem uma imagem sobre a superfície, do lado oposto e a igual distância. Essas imagens passam a ser consideradas fontes sonoras, sendo seu nível de potência corrigido em função das características de reflexão da superfície.

Para a simulação com esse programa, os dados de entrada utilizados foram: o posicionamento das vias e os valores das médias do nível sonoro equivalente (Leq em dBA), para três períodos de pico de tráfego de veículos na cidade de estudo.

Para a representação das edificações, foi admitida uma altura de 4,5 m para edifícios térreos, com adição de $3 \mathrm{~m}$ a cada pavimento superior, para edifícios de múltiplos pavimentos. Para as superfícies das fachadas foi considerado um coeficiente de absorção de 0,37 (valor padrão do software).

0 método de cálculo utilizado foi o método francês (NMPB ROUTES, 2008 apud SETRA, 2009; DUTILLEUX et al., 2008), chamado de Novo Método de Previsão do Ruído do Tráfego, recomendado pela Directive 2009/49/EC e validado por Giunta (2013) para a mesma cidade de estudo. 0 algoritmo deste método considera o cálculo do nível sonoro equivalente de longa duração (LLT) e as condições meteorológicas da zona em estudo, sendo calculado segundo a expressão:

$\mathrm{L}_{\mathrm{LT}}=10 \log \left[\mathrm{p} \cdot 10^{\mathrm{LLF} / 10}+(1-\mathrm{p}) \cdot 10^{\mathrm{LLH} / 10}\right]($ Equação 2$)$

Onde:

p: é a ocorrência das condições meteorológicas favoráveis durante a propagação do som e assume valores entre $0<\mathrm{p}<1$;
LLF: é o nível sonoro para condições meteorológicas favoráveis do local, segundo a equação 3 :

LLH: é o nível sonoro para condições meteorológicas homogêneas do local, segundo a equação 4 :

$\mathrm{L}_{\mathrm{LF}}=\mathrm{LW}-\mathrm{A}_{\mathrm{div}}-\mathrm{A}_{\mathrm{atm}}-\mathrm{A}_{\mathrm{solo}, \mathrm{F}}-\mathrm{A}_{\mathrm{dif,F}}-\mathrm{A}_{\mathrm{ref}} \quad$ (Equação 3)

$\mathrm{L}_{\mathrm{LH}}=\mathrm{LW}-\mathrm{A}_{\mathrm{div}}-\mathrm{A}_{\mathrm{atm}}-\mathrm{A}_{\mathrm{solo}, \mathrm{H}}-\mathrm{A}_{\mathrm{dif,H}}-\mathrm{A}_{\mathrm{ref}} \quad$ (Equação 4)

A variável LW é incluída nesse cálculo e representa a potência acústica associada ao fluxo de tráfego veicular, bem como à tipologia e ao tipo de pavimento da via. Os demais componentes são: Adiv, atenuação ocasionada pela divergência geométrica; Aatm, absorção pelo ar; Asolo, efeitos devido ao solo; Adif, difração e Aref, absorção das superfícies verticais nas quais o raio foi refletido no plano horizontal (SILVA, 2010).

As vias de tráfego foram simuladas como fontes lineares e, para geração do mapa de ruídos, os seguintes parâmetros de cálculo foram aplicados: receiver spacing (espaçamento entre receptores) de $10 \times 10 \mathrm{~m}$, receiver height (altura do receptor) de 1,5 m (nível do usuário) com linhas isofônicas de classe a cada 5 dBA.

Para que os valores simulados pelo modelo pudessem equivaler a valores de Leq nos diversos períodos de estudo, foram feitas simulações por períodos e limitando-se o cálculo proporcionado pelo modelo a apenas o horário específico de interesse. Essa é uma característica do programa que permite evitar a possível ponderação que poderia ser gerada pelo modelo ao integrar valores dia e noite.

Para que o modelo não fosse aplicado de forma indiscriminada, sem uma validação prévia para a região específica do estudo, foram consideradas as recomendações de Giunta (2013). Aquela pesquisa aprofundada sobre os limites e potencialidades do mesmo modelo e programa computacional aplicados à cidade de São Carlos, verificou, por meio de testes de validação e comparação entre dados medidos e simulados, que desvios nos valores preditos pelo modelo podem ser esperados, porém dentro dos limites aceitáveis. Giunta (2013) indica ainda que a configuração de entrada de dados correspondente à inserção de dados reais de Leq(A) é mais 
precisa do que a opção de inserção do fluxo de veículos como fonte sonora. A autora destacou que com a inserção de Leq(A) para gerar os mapas de ruídos, os valores simulados médios tenderam a $2 \mathrm{~dB}(\mathrm{~A})$ a menos do que os valores reais.

Para a Agência Portuguesa do Ambiente, diferenças de $\pm 2 \mathrm{~dB}(\mathrm{~A})$ entre valores simulados e medidos atendem aos limites aceitáveis. Para Silva (2010), com base nos valores apontados pelo EU-WG3 (2001), esse limite pode ser ainda menos restritivo para áreas urbanas, sendo aceitos até $\pm 4 \mathrm{~dB}(\mathrm{~A})$.

Apenas para confirmação da validação já existente, os valores simulados foram ainda comparados com os valores reais medidos e foi realizada uma análise de correspondência entre eles. Nesse caso, considerou-se que, como o Brasil ainda carece de informações e pesquisas relacionadas ao assunto, a diferença de $\pm 4 \mathrm{dBA}$ entre os valores simulados e os valores medidos em áreas urbanas pode ser adotada como limite de confiabilidade.

\section{Verificação da conformidade dos níveis sonoros com as normas vigentes}

No que diz respeito às normas brasileiras vigentes, a NBR 10.151 (ABNT, 2000) introduz definições de acústica, especifica o método para a medição do ruído e a aplicação de correções nos níveis medidos. Ainda, estabelece limites para avaliação da aceitabilidade do ruído em comunidades, por meio do Nível de Critério de Avaliação (NCA) em ambientes externos, de acordo com o tipo de zoneamento de cada área e período.

No âmbito municipal não há nenhum planejamento ou gestão de ruído específico para a cidade de São Carlos.

De acordo com o Plano Diretor da cidade, instituído pela Lei n. 13.691 de 25 de novembro de 2005, a área selecionada para estudo é considerada como Zona de Ocupação Induzida I e pertencente a uma região central e de uso misto. Ressalta-se aqui que essa área já estava consolidada antes da elaboração do Plano Diretor e, portanto, os eventuais problemas verificados não são ainda consequência direta do plano diretor atual. No entanto, pelas características da área, o Plano indica incentivo para maior ocupação de uso residencial e possibilidade de adensamento, respeitando os coeficientes máximos de ocupação (70\%) e de aproveitamento $(1,4)$. Segundo a classificação NCA, esta área é considerada como "área mista, com vocação comercial e administrativa”. Isso estabelece, pela NBR 10.151 (ABNT, 2000), um nível de ruído máximo de 60 dBA no período diurno, uma vez que este período é considerado das $7 \mathrm{~h}$ às $22 \mathrm{~h}$.

Para verificar a concordância entre níveis sonoros e normas vigentes, a aplicação de mapas de conformidade é uma alternativa de análise, como demonstrado por Costa (2011). Aquele tipo de mapa gera informações gerais, por interpolação de curvas de níveis sonoros, possibilitando a análise do entorno urbano estudado em relação aos limites legais. No entanto, esse tipo de representação não é capaz de atribuir um valor único de integração entre o ambiente interno das quadras e o ambiente ao redor das fachadas frontais, que são ambientes de características acústicas contrapostas (um protegido do ruído e outro exposto ao ruído). Assim, apesar de sua importância para a compreensão do entorno acústico, o mapa de conformidade normalmente aplicado não se configura como uma ferramenta adequada para a extração direta da informação acústica em uma escala que considere a quadra como unidade mínima de planejamento.

Por isso, para facilitar a qualificação do ambiente sonoro por meio de rápida visualização, esta pesquisa propõe que a análise de conformidade seja realizada pela ponderação desses ambientes contrapostos de quadra, gerando classes de diferenciação da contaminação sonora das quadras urbanas como unidade mínima.

\section{Proposta de classes acústicas de quadras}

Para a proposição de classes acústicas de diferenciação da contaminação sonora por quadras, foram determinados os valores médios dos níveis sonoros simulados para cada quadra. Para isso, a partir do mapa de previsão de ruídos, foram incluídos pontos de leitura (receptores) do ruído predito nas vias para cada quadra da fração estudada, na posição do ponto médio do trecho da via, correspondendo a cada face de quadra (quatro pontos no total por quadra).

O mesmo número de pontos foi incluído para leitura de dados preditos no centro das quadras, em 
posição determinada pelo afastamento de $30 \mathrm{~m}$ a partir do ponto de leitura na via, na direção do centro da quadra (Figura 2).

Com base no limite de 60 dBA aceitáveis na norma vigente para zona de uso misto, foram propostas três classes acústicas de quadras: abaixo de $55 \mathrm{dBA}$, de 55 a $60 \mathrm{dBA}$ e acima de $60 \mathrm{dBA}$. A elas foram atribuídas cores e elaborado um mapa representativo das classes de quadras.

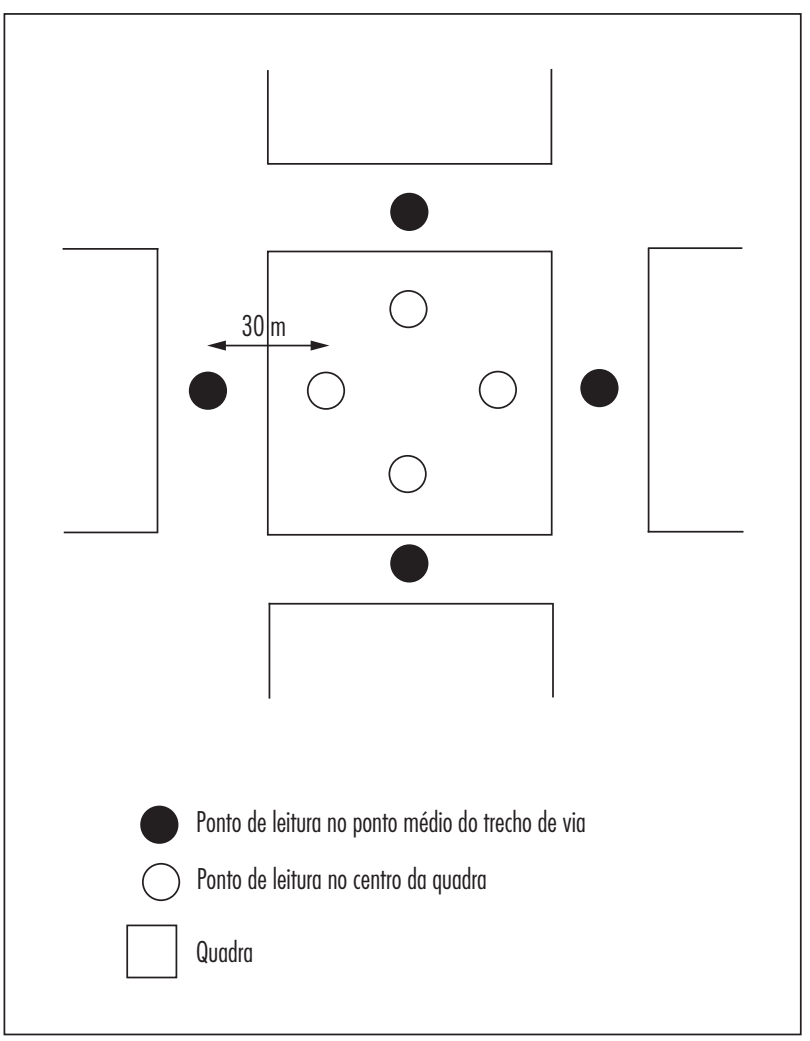

Figura 2 - Representação das posições dos pontos de leitura de valores preditos para determinação do ruído médio nas quadras

Fonte: Dados da pesquisa.

\section{Resultados}

As medições realizadas in loco para todos os períodos amostrados, de modo geral, apresentaram valores acima dos estabelecidos pela norma vigente, sendo que os Pontos 3, 6, 8, 34, 35 e 36 apresentaram valores de níveis sonoros superiores a $70 \mathrm{dBA}$ no período da manhã. Esses pontos estão situados próximos a escolas ou comércios, por conseguinte, os horários de pico coincidem com a concentração de veículos, por causa do horário de entrada e saída das escolas, que é também somado à emissão de ruídos por fontes como buzinas e vozes. No período do meio dia, os Pontos 3, 6, 8, 34 e 35 apresentaram os maiores valores, atingindo 71,95 dBA.

No período final da tarde, o maior valor de nível sonoro foi registrado no Ponto 30, local em que há um ponto de transporte coletivo e, portanto, com uma situação sujeita a frenagens e acelerações de ônibus. Por outro lado, os Pontos 1 e 26 apresentaram os menores valores (inferiores a 54,2 dBA) e estão localizados próximos a residências.

Nos demais pontos, os valores dos níveis sonoros, para todos os períodos amostrados, permaneceram entre 55,1 e 69,0 dBA, ultrapassando o limite crítico estabelecido para o conforto acústico (Gráfico 1).

Os resultados da aplicação do modelo de predição do ruído demonstraram que os valores dos níveis sonoros simulados para os horários de pico estão acima ou próximos a $60 \mathrm{dBA}$, valor limite estabelecido pela norma regulamentadora (Gráfico 2).

Para maior confiabilidade no mapa sonoro, foi realizada a validação dos resultados, a qual consistiu na comparação dos valores obtidos na simulação com os valores das medições efetuadas nos locais selecionados. Neste procedimento de validação, verificou-se que a maioria desses valores não ultrapassa $4 \mathrm{dBA}$, o que para áreas urbanas é aceitável, segundo Silva (2010). Alguns pontos excederam esse valor, como é o caso dos Pontos 25 e 33, para todos os períodos amostrados, sendo que a diferença ficou entre 4,6 e 9,6 dBA. Outros também chamaram a atenção, como, por exemplo, os Pontos 14 e 36, os quais apresentaram diferença de 9,1 dBA no período da manhã e 8,9 dBA no período do meio dia, respectivamente (Gráfico 3), bem diferente aos demais períodos. Esses são pontos que apresentam atividades de construção civil e para os quais não foi considerada, na simulação, a especificidade dessa fonte sonora, em virtude da dificuldade de se estabelecer isoladamente, no local, a emissão dessa fonte.

De uma forma geral, a maioria dos valores simulados a partir da inserção dos dados reais tendeu a alcançar níveis mais baixos do que os medidos, como havia sido apontado por Giunta (2013).

Na análise de correlação entre os dados medidos e os simulados (Gráfico 4), os valores de $\mathrm{R}^{2}$ para todos os períodos foram considerados elevados, o que confirma a validação dos resultados. 


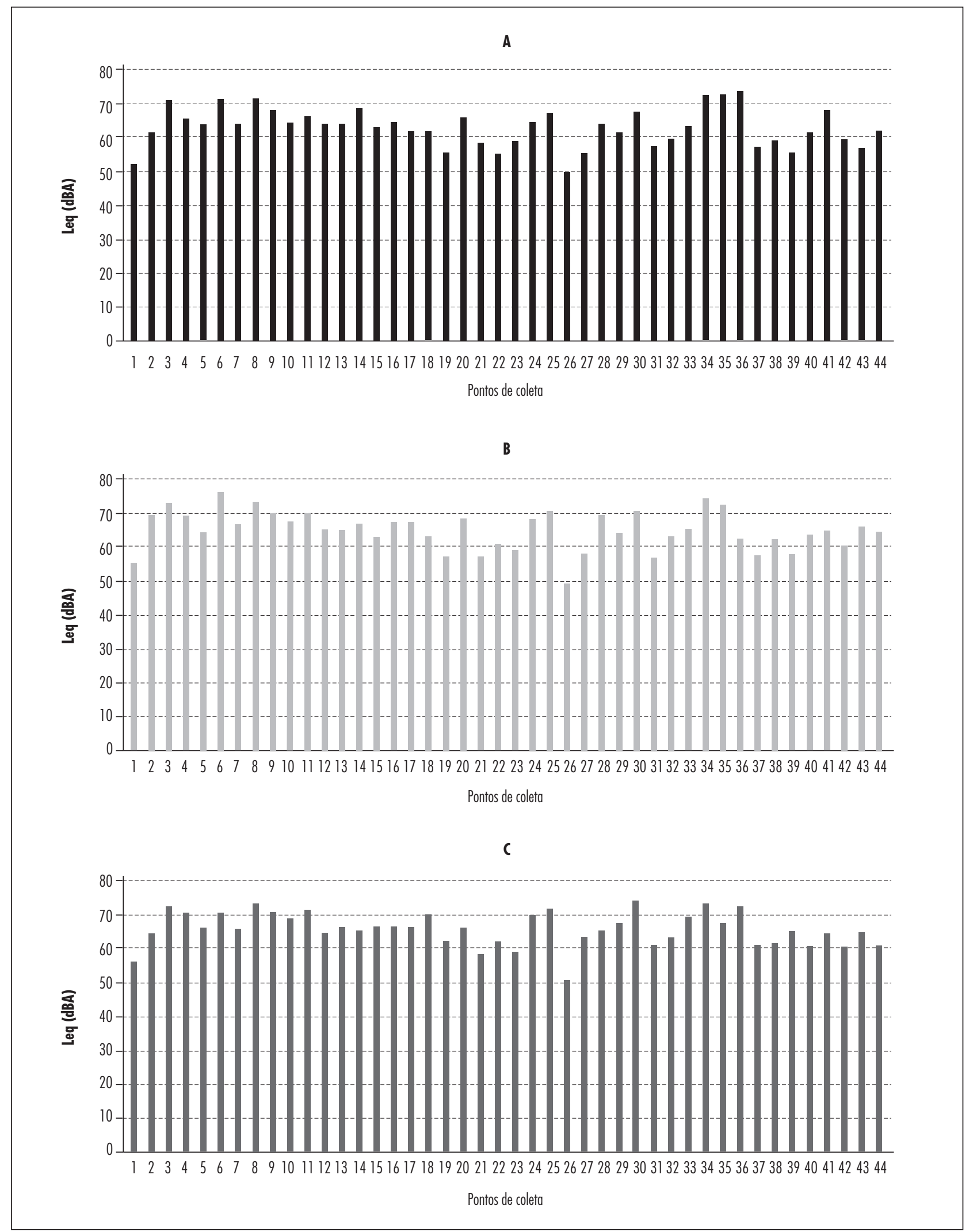

Gráfico 1 - Valores de Leq (dBA) medidos in loco em horários de pico para os períodos "A" (7-8h), "B" (12-13h) e "C" (17h30-18h30), na cidade de São Carlos (São Paulo, Brasil)

Fonte: Dados da pesquisa. 


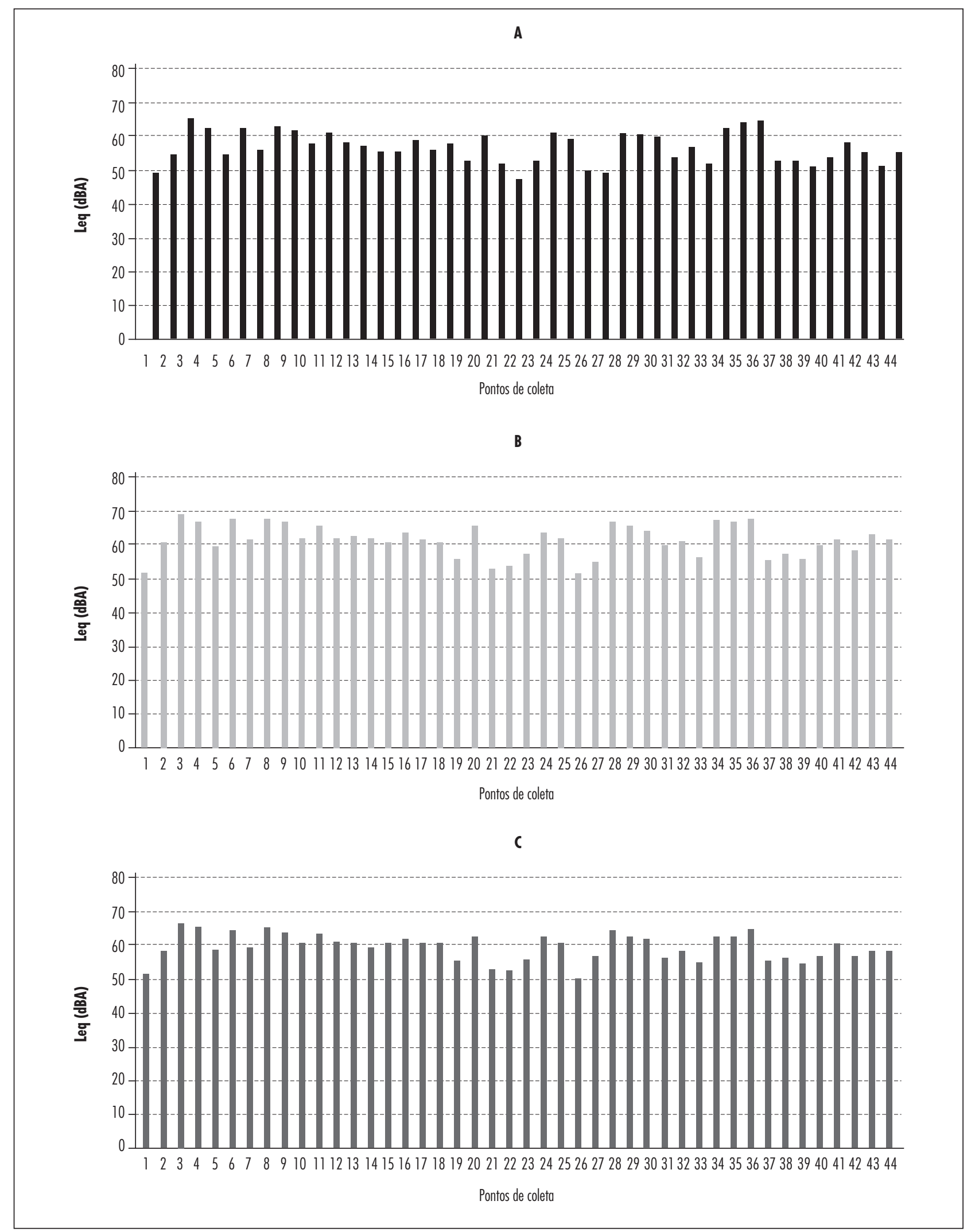

Gráfico 2 - Valores de Leq (dBA) simulados para os horários de pico nos períodos "A" (7h-8h), "B" (12h-13h) e "C" (17h30-18h30), na cidade de São Carlos (São Paulo, Brasil)

Fonte: Dados da pesquisa. 


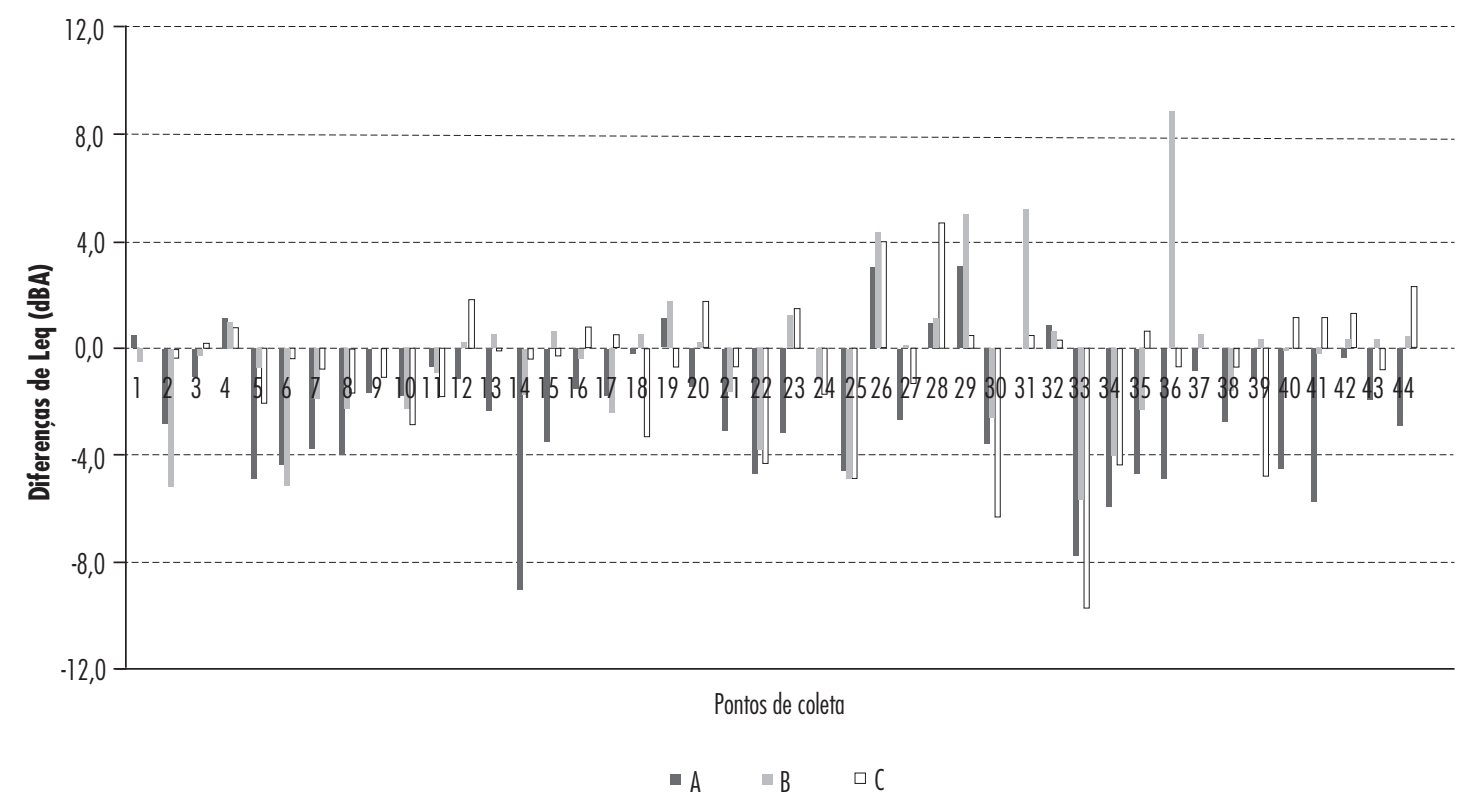

Gráfico 3 - Diferença entre os valores de Leq (dBA) simulados e medidos para os horários de pico nos períodos "A" (7h-8h), "B" (12h-13h) e "C" (17h30-18h30), na cidade de São Carlos (São Paulo, Brasil) Fonte: Dados da pesquisa.

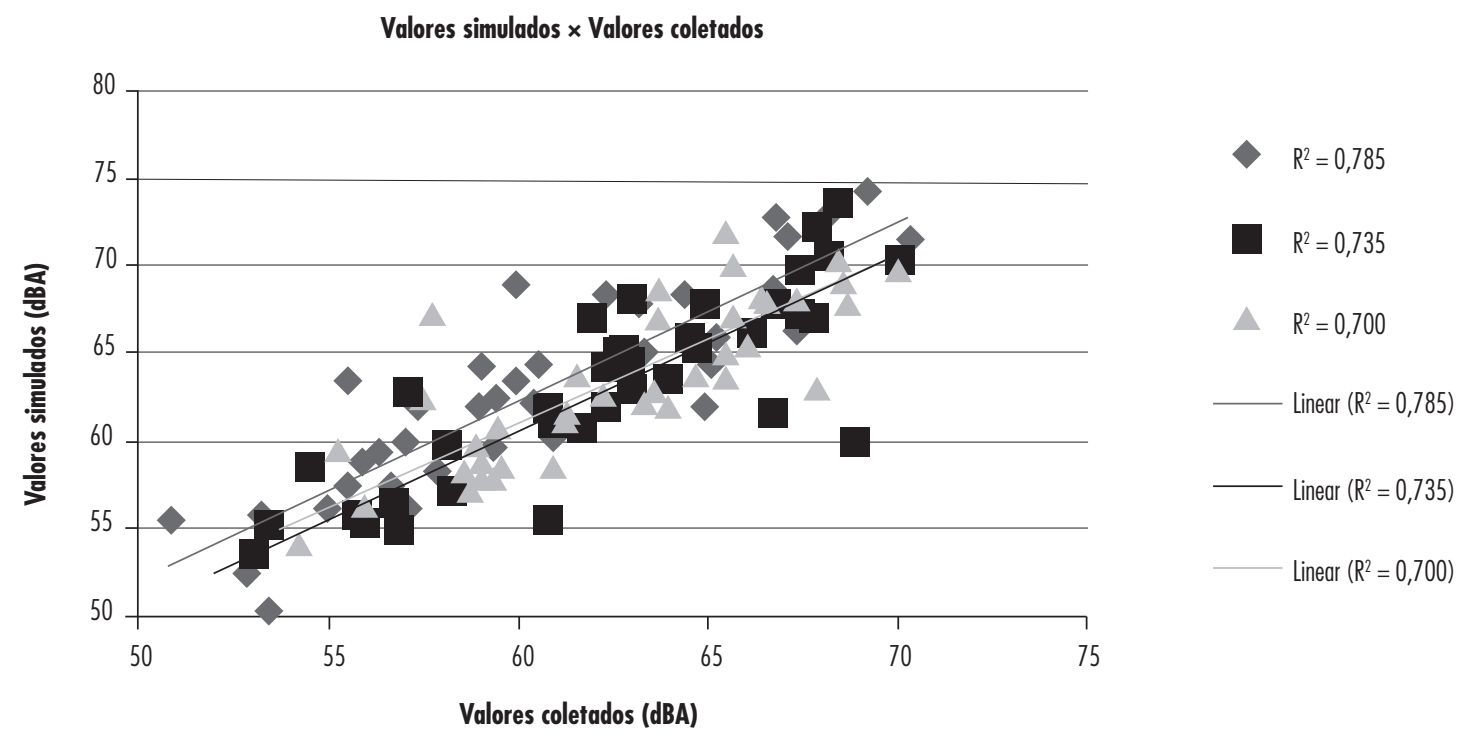

Gráfico 4 - Análise de correspondência entre os valores obtidos in loco e os simulados para os horários de pico nos períodos amostrados: "A" (7h-8h), "B" (12h-13h) e "C" (17h30-18h30)

Fonte: Dados da pesquisa. 
0 mapa sonoro para a região de estudo, simulado a uma altura de 1,5 m em relação ao solo, está apresentado na Figura 3, no qual pode-se visualizar que as áreas de maiores níveis sonoros estão localizadas nas vias que apresentam maior fluxo de veículos, como é o caso das ruas Carlos Botelho e XV de Novembro. Neste mapa, também é possível analisar o comportamento acústico no interior das quadras, as quais apresentam níveis sonoros menores que os encontrados nas vias. Este fato decorre do distanciamento destas regiões em relação à fonte sonora (tráfego de veículos), como também ao fator destas áreas estarem protegidas pelas edificações, que agem como barreiras acústicas, atenuando os níveis de pressão sonora.

Nessas quadras não há diferenciação de pavimentação e, portanto, os níveis alcançados de ruídos podem ser atribuídos em sua grande parte ao fluxo de tráfego, aos maiores limites de velocidade permitidos nos eixos principais e suas interações com todo o ambiente construído do entorno. Assim, quando é observada a Figura 3, verifica-se que as quadras que apresentam o maior fluxo de veículos e maior velocidade limite $(40 \mathrm{~km} / \mathrm{h})$ tendem a atingir níveis de ruídos nos horários de pico mais elevados do que os níveis legais.

Com o resultado da proposta de classificação das quadras por faixa de ruído médio (Figura 4), constatou-se que dentro da zona de ocupação estudada existem quadras mais críticas, apresentando níveis de ruído que demonstram a incompatibilidade entre uma proposta de adensamento e o conforto acústico. São quadras que apresentam um entorno com maior fluxo de veículos em meio às demais, incluindo-se, dentre elas, uma quadra de área verde e lazer.

Observa-se que apenas sete dessas quadras possuem, de fato, maior aceitação acústica para o adensamento.

0 adensamento representa o aumento no fluxo de veículos e, consequentemente, intensificação da fonte sonora. Portanto, as diretrizes gerais de ocupação nessa área deveriam ser mais restritivas em relação às quadras cuja classe corresponde àquela acima de $60 \mathrm{dBA}$. Mesmo as quadras que se encontram no limite, entre 55 e $60 \mathrm{dBA}$, indicam que o adensamento nessa área deve ser bastante cauteloso.

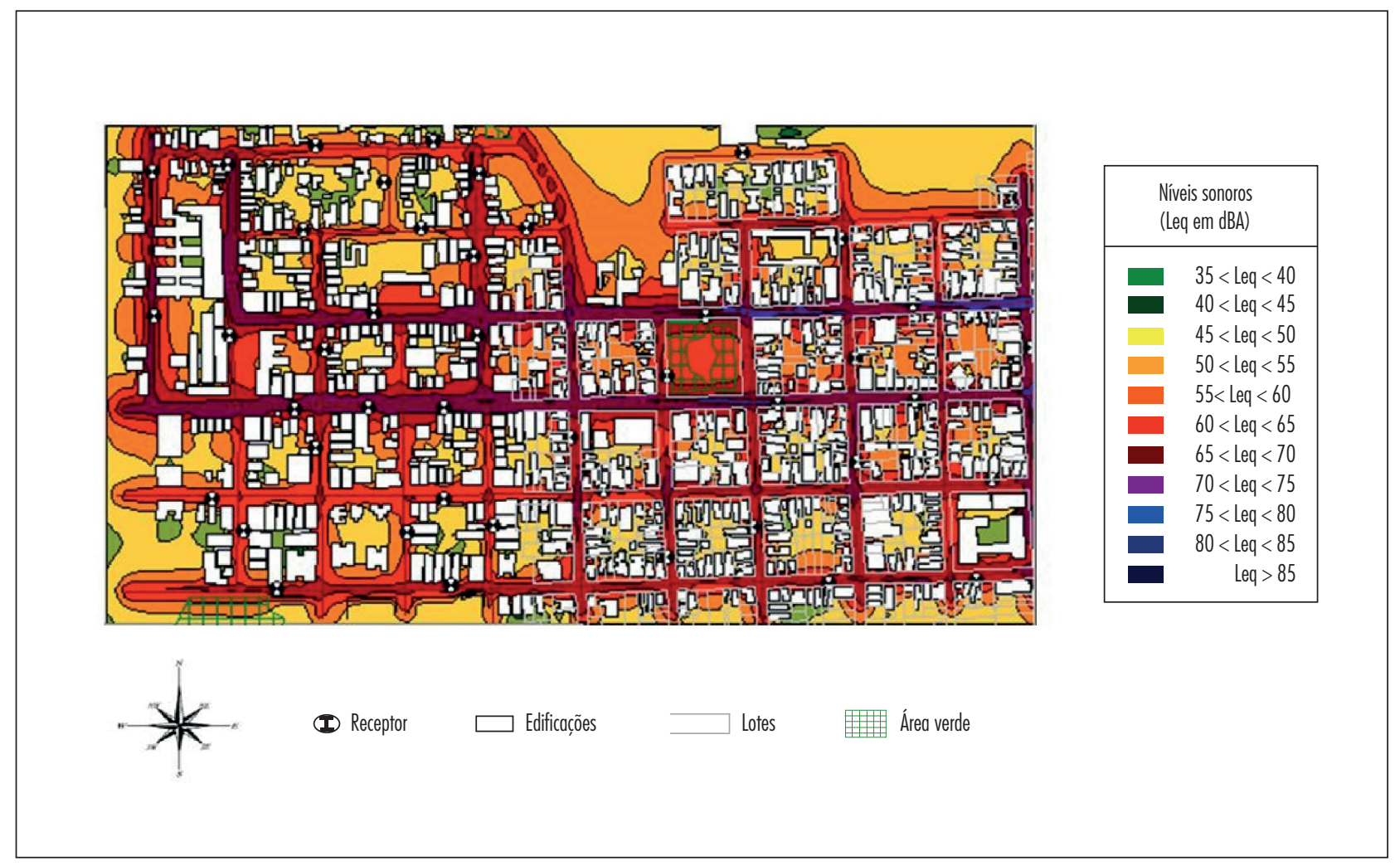

Figura 3 - Mapa de ruído para o período amostrado na cidade de São Carlos, SP, Brasil Fonte: Dados da pesquisa. 

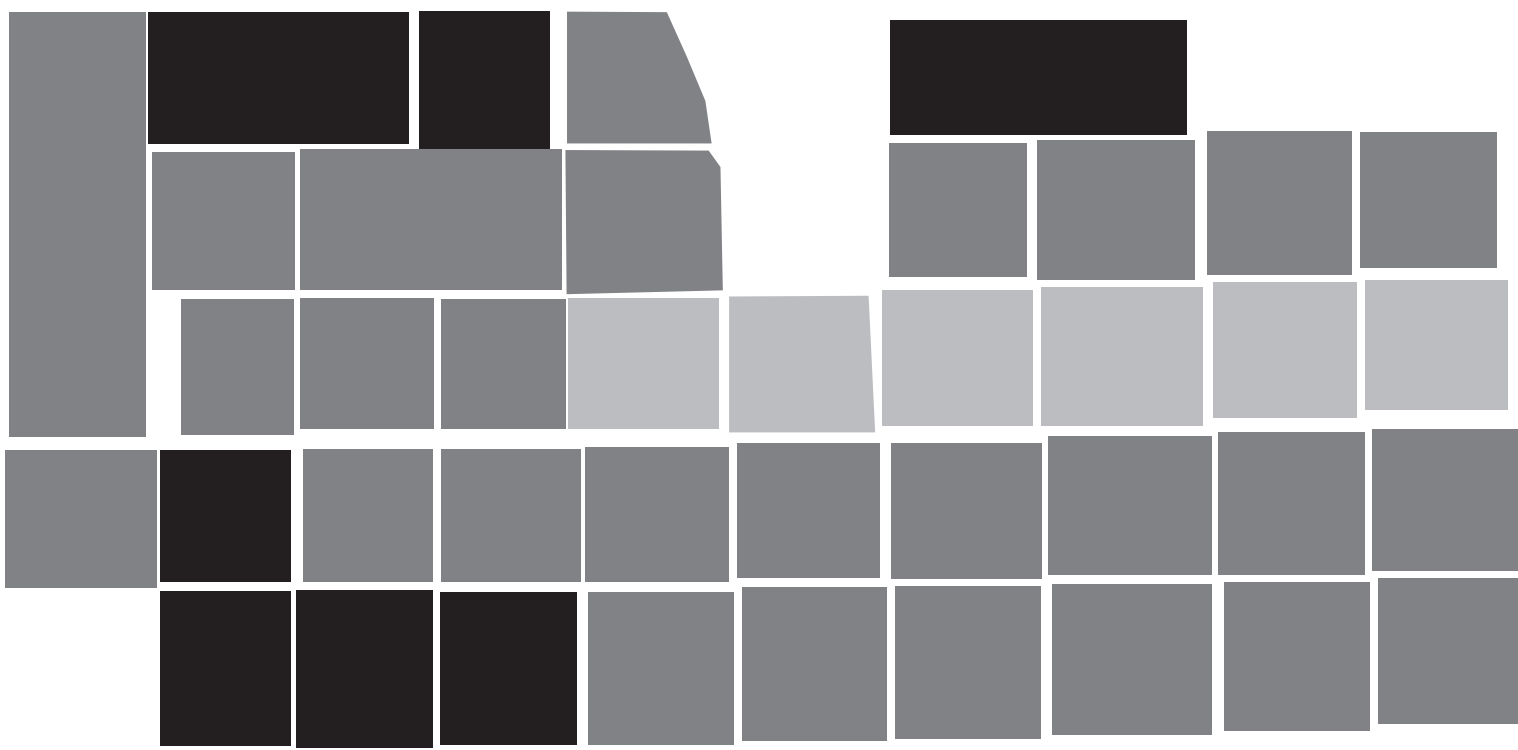

abaixo de $55 \mathrm{~dB}$

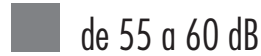

acima de $60 \mathrm{~dB}$

Figura 4 - Mapeamento das classes acústicas de quadras urbanas para horários de pico na cidade de São Carlos (São Paulo, Brasil)

Fonte: Dados da pesquisa.

\section{Discussão}

O problema da poluição sonora estende-se nas mais diversas cidades brasileiras em função da falta de planejamento urbano e da negligência diante dos cuidados relacionados aos sons emitidos, não existindo de fato um combate mais efetivo.

Nas cidades de grande porte, esse problema é intensificado graças às múltiplas fontes sonoras, principalmente ao intenso tráfego viário. Isso pode ser constatado em pesquisas realizadas em Curitiba (CANTIERI et al., 2010), onde os valores médios atingem 81,9 dBA, representando 16,9 dBA acima do estabelecido, e no Rio de Janeiro, onde Pinto e Mardones (2009) encontraram valores superiores a $65 \mathrm{dBA}$.

Os níveis sonoros ainda são considerados incômodos nas cidades de médio porte, como se pode constatar nos trabalhos realizados em Sorocaba (COSTA; LOURENÇO, 2010), Taubaté (BRITO; SINDER, 2009), Bauru (SOUZA; GIUNTA, 2011) e em algumas cidades de Minas Gerais (VALADARES et al., 2007). Um diferencial das cidades de médio e pequeno porte brasileiras é que, apesar de muitas vezes apresentarem esse problema, demonstram um potencial mais promissor ao tratamento preventivo dos ruídos do que as grandes cidades.

No caso da cidade de São Carlos, como pode ser avaliado neste trabalho, a maioria dos níveis sonoros alcançados é considerada elevada, uma vez que ultrapassa o limite estabelecido pelas normas vigentes.

A análise do mapa sonoro da cidade foi capaz de identificar áreas críticas e preservadas, mostrando também que esta tecnologia é um excelente mecanismo para lidar com o problema da poluição sonora, apresentando também subsídios para tomadas de decisão e aplicação no planejamento urbano.

Com relação à simulação computacional, o software CADNA-A demonstrou-se eficaz para avaliação do impacto sonoro, corroborando com outros trabalhos que também utilizaram essa mesma ferramenta computacional (PINTO, MARDONES, 2009; ARANA; SAN MARTIN, R.; SAN MARTIN, M.L., 2010; FORASTER et al., 2011). 
A análise de contaminação sonora por pontos receptores permite a identificação dos níveis em pontos urbanos específicos, o que auxilia na tomada de decisão para a prevenção e controle do ruído. Porém, existe uma lacuna entre a escala pontual propiciada pelo mapeamento sonoro e a escala das diretrizes determinadas pelos municípios para cada zona de ocupação. Se por um lado as decisões urbanas não podem ser tomadas por questões apenas pontuais, por outro, a consideração das normas vigentes, sob o ponto de vista da escala da zona de ocupação como um todo, acaba por generalizar diretrizes de adensamento que podem intensificar a contaminação sonora já existente. Por essa razão, a abordagem por quadras, após o mapeamento e a previsão de níveis de pressão sonora do entorno, pode ser uma solução eficaz, permitindo evidenciar aquelas quadras que não comportam mais adensamento ou intensificação do fluxo de veículos, no que diz respeito à contaminação sonora. Assim, é possível estabelecer ações mais restritivas para o controle e prevenção do ruído.

A proposta de serem estabelecidas classes por quadras (abaixo do limite, no limite e acima do limite), fixando-se uma faixa limite, ou seja, $5 \mathrm{dBA}$ a menos do que o limite da norma até o valor limite estabelecido pela norma, pode ser extrapolada para outros tipos de zonas e usos. Isso permite uma comparação entre as normas vigentes para aquele uso e a situação de ruído ocorrida em qualquer zona urbana.

\section{Conclusões}

O mapeamento do ruído do ambiente exterior para a situação de pico de tráfego, em uma região da cidade considerada como uso misto, constatou que os níveis sonoros, tanto in loco como os simulados, estão acima do permitido pela legislação, o que reflete um alerta para o conforto sonoro ambiental.

De forma geral, os resultados da comparação dos valores dos níveis sonoros medidos e dos simulados foram satisfatórios, não ultrapassando $4 \mathrm{dBA}$, podendo-se inferir que esse modelo experimental se encontra validado para a região de estudo.

A criação das classes de ruído por quadra é a contribuição mais significativa deste estudo, pois se torna uma ferramenta de grande utilidade para os responsáveis pelo ordenamento e planejamento do território municipal, em articulação com os responsáveis pela gestão ambiental. 0 mapeamento dessas classes permitiu evidenciar quadras para as quais ações de controle devem ser tomadas para a garantia da qualidade acústica do local.

\section{Agradecimentos}

Os autores agradecem à Coordenação de Aperfeiçoamento de Pessoal de Nível Superior (CAPES) e ao Conselho Nacional de Desenvolvimento Científico e Tecnológico (CNPq) pelo suporte técnico e financeiro disponibilizados.

\section{Referências}

ASSOCIAÇÃO BRASILEIRA DE NORMAS TÉCNICAS ABNT. NBR 10151: acústica - avaliação do ruído em áreas habitadas, visando o conforto da comunidade procedimento. Rio de Janeiro, 2000.

ASSOCIAÇÃO BRASILEIRA DE NORMAS TÉCNICAS ABNT. NBR 7731: guia para execução de serviços de medição de ruído aéreo e avaliação de seus efeitos sobre o homem. São Paulo, 1983.

ASSOCIAÇÃO BRASILEIRA DE NORMAS TÉCNICAS ABNT. NBR 10152: níveis de ruído para o conforto acústico. Rio de Janeiro: ABNT, 1987.

ARANA, M.; SAN MARTIN, R.; SAN MARTIN, M.L. Strategic noise map of a major road carried out with two environmental prediction software packages. Environmental Monitoring and Assessment, n. 163, p. 503-513, 2010.

BRASIL. Conselho Nacional do Meio Ambiente. Resolução Conama n. 001, de 8 de março de 1990. Dispõe sobre critérios e padrões de emissão de ruídos. Brasília, DF, 8 mar. 1990a. Disponível em: <http://www.mma.gov.br/ port/conama/legiabre.cfm?codlegi=98>. Acesso em: 10 jul. 2012.

BRASIL. Conselho Nacional do Meio Ambiente. Resolução Conama n. 002, de 08 de março de 1990. Dispõe sobre o Programa Nacional de Educação e Controle da Poluição Sonora - "SILÊNCIO". Brasília, DF, 8 mar. 1990b. Disponível em: <http://www.mma.gov.br/port/conama/ legiabre.cfm?codlegi=99>. Acesso em: 10 jul. 2012. 
BRASIL. Conselho Nacional do Meio Ambiente. Resolução Conama n. 020, de 8 de dezembro de 1994. Institui o Selo Ruído, como forma de indicação do nível de potência sonora, de uso obrigatório para aparelhos eletrodomésticos. Brasília, DF, 8 dez. 1994. Disponível em: <http://www.mma.gov.br/port/conama/legiabre. cfm?codlegi=161>. Acesso em: 10 jul. 2012.

BRITO, L. A. P. F.; SINDER, V. Determinação do nível de pressão sonora das principais vias públicas da região central de Taubaté. In: ENCONTRO NACIONAL DE CONFORTO NO AMBIENTE CONSTRUÍDO, 10. E ENCONTRO LATINOAMERICANO DE CONFORTO NO AMBIENTE CONSTRUíDO, 5., 2009, Natal. Anais... Natal: Antac, 2009.

CANTIERI, E. et al. Elaboração de um mapa de ruído para a região central da cidade de Curitiba - PR. Produção on-line, v. 10, n. 1, 2010. Disponível em: <http://producaoonline.org.br/rpo/article/view/239/576>. Acesso em: 16 out. 2013.

CARVALHO, L. C. Aplicação da legislação sobre ruído à escala do aglomerado urbano. In: ENCONTRO NACIONAL SOBRE QUALIDADE E INOVAÇÃO NA CONSTRUÇÃO (QIC 2006). 2006, Lisboa. Anais... Disponível em: <https://sapientia.ualg.pt/bitstream/10400.1/194/1/CARApl.pdf>. Acesso em: 10 dez. 2012.

COSTA, S. B.; LOURENÇO, R. W. Geoprocessing applied to the assessment of environmental noise: a case study in the city of Sorocaba, São Paulo, Brazil. Environmental Monitoring and Assessment, n. 172, p. 329-337, jan. 2010.

COSTA, D. A. Previsão dos ruídos ambientais no setor noroeste, Brasília - DF. Dissertação (Mestrado em Planejamento e Gestão Ambiental) - Universidade Católica de Brasília, Brasília, 2011.

DATAKUSTIK. CADNA Manual V3.4. Greifenberg: Datakustik GMBH, 2005.

DUTILLEUX, G. et al. NMPB-ROUTES-2008: the revision of the French method for road traffic noise prediction. Acta Acustica united with Acustica, v. 96, n. 3, p. 452462, 2010.

EUROPEAN COMMUNITIES. Directive 2002/49/EC of the European Parliament and of the Council, of 25 June 2002. Relating to the assessment and management of environmental noise. Official Journal of the European Communities, July 18 2002. Disponível em: <http://eur-lex.europa.eu/LexUriServ/LexUriServ.do?uri=0J:L:2002 :189:0012:0025:EN:PDF>. Acesso em: 16 out. 2013.
FORASTER, M. et al. Local determinants of road traffic noise levels versus determinants of air pollution levels in a Mediterranean city. Environmental Research, v. 111, p. 177-183, 2011.

GIUNTA, M.B. Análise de modelagem de precisão acústica e mapeamento sonoro para a cidade de São Carlos SP. 2013. Dissertação (Mestrado em Engenharia Urbana) Universidade Federal de São Carlos, São Carlos, 2013.

GUEDES, C. M.; BERTOLI, S. R. Forma urbana: um indicativo de sua influência no ambiente sonoro no bairro Jardins em Aracaju (SE). In: ENCONTRO NACIONAL DE CONFORTO NO AMBIENTE CONSTRUÍDO, 8. E ENCONTRO LATINOAMERICANO DE CONFORTO NO AMBIENTE CONSTRUÍDO, 4., 2005, Maceió. Anais... Maceió: Antac, 2005.

INSTITUTO BRASILEIRO DE GEOGRAFIA E ESTATÍSTICA IBGE. Indicadores sociais municipais: uma análise dos resultados do universo do Censo Demográfico 2010. 2011. Disponível em: <http://www.ibge.gov.br/home/ estatistica/populacao/censo2010/indicadores_sociais_ municipais/indicadores_sociais_municipais.pdf>. Acesso em: 16 out. 2013.

INTERNATIONAL ORGANIZATION FOR STANDARDIZATION. ISO 1996/2. Acoustics: description, measurement and assessment of environmental noise - part 2: determination of environmental noise levels, 2007/2. Genebra, 2007.

MARDONES, M. D. M. Mapeamento dos níveis de ruído em Copacabana, Rio de Janeiro, através de simulação computacional. 2009. Dissertação (Mestrado em Engenharia Mecânica) - Universidade Federal do Rio de Janeiro, Rio de Janeiro, 2009.

MENDONÇA, A. B. D. et al. Medições acústicas: variação da geometria da via urbana e tempo de amostragem. In: SIMPÓSIO DE PÓS GRADUAÇÃO EM ENGENHARIA URBANA, 3., 2012, Maringá. Anais... Maringá: Universidade Estadual de Maringá, 2012. Disponível em: <http://www.eventos.uem.br/index.php/simpgeu/simpgeu/paper/view/932/579>. Acesso em: 16 out. 2013.

MORAES, E. M. L. Que é e como se faz um mapa estratégico de ruído. In: ENCONTRO NACIONAL DE CONFORTO NO AMBIENTE CONSTRUÍDO, 9. E ENCONTRO LATINOAMERICANO DE CONFORTO NO AMBIENTE CONSTRUíDO, 5., 2007, Ouro Preto. Anais... Ouro Preto: Antac, 2005. 
MORAES, E. et al. Mapa de ruídos da zona comercial de Belém, uma parcela do mapa de ruídos da cidade de Belém - Brasil. Bilbao: Tecni Acustica, 2003.

PINTO, F. A. N. C.; MARDONES, M. D. M. Noise mapping of densely populated neighborhoods: example of Copacabana, Rio de Janeiro - Brazil. Environmental Monitoring and Assessment, v. 155, p. 309-318, 2009.

SANTOS, L. C.; VALADO, F. The municipal noise map as planning tool. In: ACÚSTICA, 2004, Guimarães. Proceedings... Guimarães: Universidade do Minho, 2004. Paper ID:162.

SECRETARIA MEIO AMBIENTE E CONTROLE URBANO DE FORTALEZA - SEMAM. Carta acústica. 2011. Disponível em: <http://www.fortaleza.ce.gov.br/carta-acustica?option=com_content\&task=view\&id=59> Acesso em: 30 jan. 2013.

SERVICE D'ETUDES SUR LES TRANSPORTS, LES ROUTES ET LEURS AMENAGEMENTS - SETRA. Road noise Prediction, 2: NMPB 2008 - Noise propagation computation method including meteorological effects. 2009. Disponível em:<http://www.setra.developpement-durable.gouv.fr/>. Acessado em: 10 dez. 2012.

SILVA, A. M. C. Mapa de ruído do bairro "Vila Universitária" Bauru, Brasil - Situação de pico. 2010. Dissertação (Mestrado em Engenharia) — Universidade do Minho, Braga, 2010.
SOUZA, L. C. L.; GIUNTA, M. B. Urban indices as environmental noise indicators. Computers, Environment and Urban Systems, v. 35, n. 5, p. 421-430, 2011.

VALADARES, V. M. et al. Considerando a atmosfera acústica na elaboração de planos diretores. In: ENCONTRO NACIONAL DE CONFORTO NO AMBIENTE CONSTRUÍDO, 9. E ENCONTRO LATINOAMERICANO DE CONFORTO NO AMBIENTE CONSTRUÍDO, 5., 2007, Ouro Preto. Anais... Ouro Preto: Antac, 2005.

WOLRD HEALTH ORGANIZATION - WHO. Burden of disease from environmental noise: quantification of healthy life years lost in Europe. Copenhagen: WHO Regional Office for Europe, 2011. Disponível em: <http:// www.who.int/quantifying_ehimpacts/publications/ e94888.pdf>. Acessado em 8 ago. 2012.

Recebido: 28/06/2013

Received: 06/28/2013

Aprovado: 19/09/2013

Approved: 09/19/2013 